\title{
Direct Visualization of Lithium Intercalation in Spinel Iron Oxide by In-Situ Bright-Field Scanning Transmission Electron Microscopy
}

\author{
Kai $\mathrm{He}^{1,2}$, Jing $\mathrm{Li}^{1}$, Qingping Meng ${ }^{3}$, Eric A. Stach ${ }^{1}$, Yimei $\mathrm{Zhu}^{3}$ and Dong $\mathrm{Su}^{1}$ \\ 1. Center for Functional Nanomaterials, Brookhaven National Laboratory, Upton, NY 11973 \\ 2. Department of Materials Science and Engineering and NUANCE Center, Northwestern University, \\ Evanston, IL 60208 \\ 3. Condensed Matter Physics and Materials Science Department, Brookhaven National Laboratory, \\ Upton, NY 11973
}

In situ transmission electron microscopy (TEM) is a fast-growing area and has attracted tremendous attention in diverse scientific research spanning from materials science to chemistry and biology [1]. Specifically, the advancement of in situ TEM in chemically reactive environments has enabled the direct real-time observation of electrochemical reactions in electrode materials for lithium ion batteries [2-4]. Prior in situ studies have mostly focused on the electrodes that react with lithium through alloying or conversion reactions, since these processes involve obvious structural and chemical changes that can be readily captured by direct imaging and spectroscopy methods on the nanoscale $[2,3]$. On the other hand, the lithium intercalation process as the predominant mechanism of the present battery technology, however, is hard to be tracked due to its insignificant volume change [4]. Here, we present the development of a new strain-sensitive bright-field scanning transmission electron microscopy (BFSTEM) and its implementation of imaging the intercalation reaction during the lithiation of a single magnetite $\left(\mathrm{Fe}_{3} \mathrm{O}_{4}\right)$ nanocrystal.

$\mathrm{Fe}_{3} \mathrm{O}_{4}$ has been widely considered as a promising electrode material because of its ample abundance, non-toxicity, low cost, and high theoretical capacity. We use $\mathrm{Fe}_{3} \mathrm{O}_{4}$ nanoparticles $(\sim 80 \mathrm{~nm}$ truncated octahedrons, Figure 1a) with the spinel structure that can accommodate guest $\mathrm{Li}^{+}$ions at the vacant octahedral 16c positions and repel $\mathrm{Fe}^{3+}$ cations from tetrahedral $8 \mathrm{a}$ to the nearby octahedral $16 \mathrm{c}$ sites (Figure 1b). This is an intercalation reaction to cause phase transformation from spinel $\mathrm{Fe}_{3} \mathrm{O}_{4}$ to rocksalt $\mathrm{LiFe}_{3} \mathrm{O}_{4}$ (Figure 1c) [5]. Further lithiation will lead to thorough structure destruction to form a composite of metallic $\mathrm{Fe}$ and amorphous $\mathrm{Li}_{2} \mathrm{O}$ through a conversion reaction (Figure 1d), like in many other transition metal oxides [2]. The entire process of two-step lithiation has also been confirmed by the in situ electron diffraction (Figure 1e-g).

Besides the HRTEM imaging to monitor the atomic structure reconstruction, we also demonstrated a new approach to directly visualize the Li intercalation within the entire single nanoparticle using in situ BF-STEM on the nanoscale (Figure 2). Comparison between simultaneously acquired HAADF-STEM and BF-STEM images (Figure 2a, b) clearly illustrates the visually distinguishable phase boundary by the contrast arising from the coherent electrons scattered to lower angles, which are more sensitive to a small strain $(\sim 1 \%$ in this case) induced by negligible volume expansion due to Li insertion. The timeresolved BF-STEM images explicitly show the kinetic evolution of both intercalation and conversion pathways at non-equilibrium conditions. These findings not only elucidate the atomistic mechanism for the two-step lithiation in spinel structured oxides, but also demonstate a valid methodology for investigating lithium insertion in other intercalation electrode materials or probing strain-sensitive phase transformations in general [6]. 
References:

[1] H Zheng, YS Meng, and Y Zhu, MRS Bulletin 40, (2015) 12.

[2] JY Huang et al, Science 330, (2010) 1515.

[3] K He et al, Nano Lett. 15, (2015) 1437.

[4] Y Wen, K He et al, Nature Commun. 5, (2014) 4403.

[5] MM Thackeray, J. Am. Ceram. Soc. 82, (1999) 3347.

[6] The authors acknowledge funding support from U.S. Department of Energy, Office of Basic Energy Sciences, under Contract Number DE-SC-00112704.
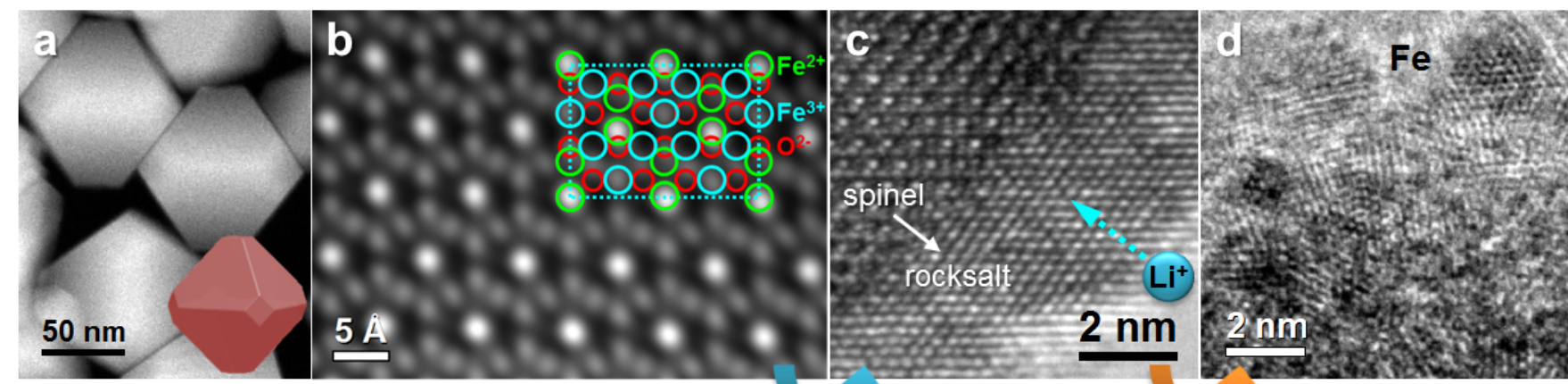

Pristine $\left(\mathrm{Fe}_{3} \mathrm{O}_{4}\right)$
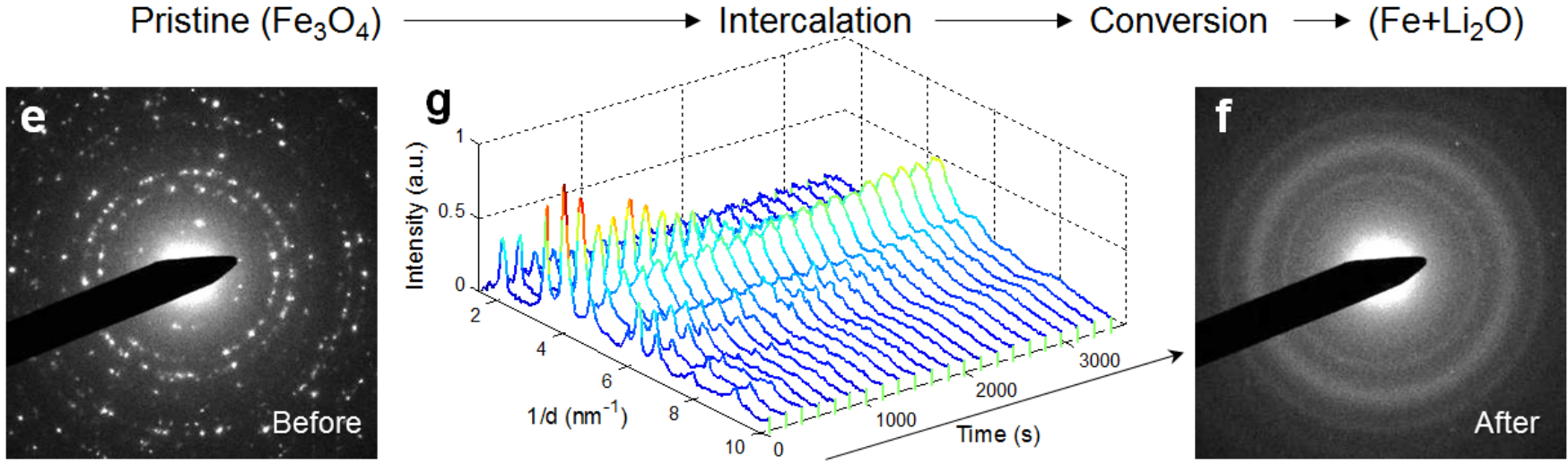

Figure 1. Structural evolution of $\mathrm{Fe}_{3} \mathrm{O}_{4}$ upon lithiation. (a) $\mathrm{Fe}_{3} \mathrm{O}_{4}$ nanoparticles in truncated octahedral shape showing (b) spinel structure at the pristine state. (c) Structural reconstruction from spinel to rocksalt as lithiated to form $\mathrm{LiFe}_{3} \mathrm{O}_{4}$. (d) Composite of metallic Fe nanocrystallites and amorphous $\mathrm{Li}_{2} \mathrm{O}$ after conversion reaction. Electron diffraction patterns (e) before, and (f) after a full lithiation, with (g) time-resolved profiles retrieved from in situ electron diffraction to confirm the two-step lithiation.
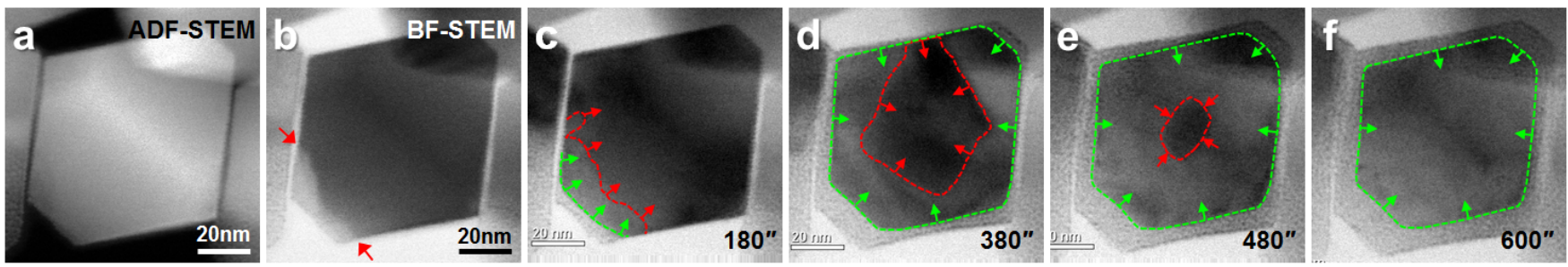

Figure 2. Time-lapsed in situ BF-STEM imaging of a single $\mathrm{Fe}_{3} \mathrm{O}_{4}$ nanoparticle during lithiation. (a) ADF-STEM and (b) BF-STEM images of a same particle showing explicit reaction front in BF-STEM. (c-f) Snapshots at a series of lithiation time to illustrate kinetic evolution of intercalation (red) and conversion (green) reaction pathways. 